\title{
COLCHICINA: INTOXICAÇÃO E ÓBITO, RELATO DE CASO.
}

\section{COLCHICINE: INTOXICATION AND DEATH, CASE REPORT}

\author{
GABRIEL,P.M.K.C ${ }^{1,2 *}$; GABRIEL,M.M²; ESCORSIN NETO, J'; LOPES, $M^{1,2}$.
}

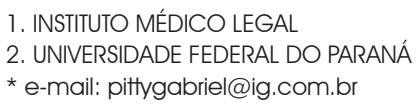

* e-mail: pittygabriel@ig.com.br

Recebido em: 02/2004

Aprovado em: 05/2004

RESUMO

B. I., de 67 anos, foi internado em um hospital no interior do Estado do Paraná, apresentando dor abdominal, vômitos e diarréia intensa, vindo a falecer após 4 h de internamento. Após investigação do médico sanitarista, constatou-se que a vítima havia começado tratamento com cápsulas manipuladas com o princípio ativo "colchicina". Análise por cromatografia líquida de alta resolução (HPLC) indicou positividade para colchicina nas cápsulas, com concentração média de 63,48mg e negativo no material biológico da vítima. A intoxicação por colchicina foi confirmada através dos exames anátomo patológico, sintomatologia apresentada no período de internamento e a alta dosagem de colchicina encontrada nas cápsulas manipuladas e ainda disponíveis. Palavras-chave: Colchicum autumnale, colchicina, gota

\section{ABSTRACT}

B. I., sixty-seven years old, was taken to a hospital with abdominal cramps, vomiting and intense diarrhoea; he died four hours after being admitted in the emergency ward. After some medical investigation, it became known that the patient had started a treatment with colchicine in capsules prepared by a pharmacist. The capsules and the autopsy materials were analysed by High Performance Liquid Chromatography (HPLC) and the analytical results for the capsules were positive for colchicine with mean concentration of $63,48 \mathrm{mg} / \mathrm{capsule}$; the analytical results for the autopsy materials were negative for colchicine. The fatal colchicine intoxication was confirmed by the anatomo-pathological exams carried out in the autopsy materials, by the sintomatology presented by the patient during his staying in the hospital and by the high concentration of colchicine found in the still remaining capsules.

Keywords: Colchicum autumnale, colchicine, gout

\section{INTRODUÇÃO}

A colchicina, principal alcalóide do Colchicum autumnale, é um medicamento antinflamatório clássico para o tratamento das crises agudas da gota e de sua prevenção (GOLDFRANK et al, 1994; KINTZ et. al, 1997; ELLENHORN, 1997; GONZÁLEZ ANGLADA, 1998;). Segundo BATISTUZZO et al (2000), a colchicina também tem outras indicações como na: amiloidose, dermatite herpetiforme, dermatomiosites, esclerodermia, psoríase, púrpura trombocitopênica idiopática, quelóides e vasculite crotizante. Quando utilizada determina secundarismo; principalmente para o lado digestivo, com náusea, vômito, diarréia e dor abdominal; controlável com a diminuição da dose ou suspensão da mesma (REYNOLDS, 1989).

A intoxicação por colchicina é rara mas potencialmente grave (BAUD, 1995; MEGARVANE, 2000), com falência cardíaca, respiratória, hepática, neurológica e hematológica (GOLDFRANK et al, 1994; KINTZ et. Al, 1997; MEGARVANE, 2000); mas há relato na literatura de intoxicação e óbito por ingestão de flores de Colchicum autumnale, sem que houvesse alteração dos valores normais nos exames hematológicos (DANEL, 1994). É um potente tóxico gastrointestinal (GOLDFRANK et al, 1994; KINTZ et. al, 1997); apresentando grau de severidade e mortalidade intimamente relacionados com a dose ingerida, de acordo com a Tabela 1. (GOLDFRANK et al, 1994; LEIKIN, 1998; MEGARVANE, 2000). 
TABELA 1: DOSE INGERIDA DE COLCHICINA (mg/kg) X MORTALIDADE (\%)

\begin{tabular}{cc}
\hline dose supostamente ingerida & mortalidade \\
\hline $0,5 \mathrm{mg} / \mathrm{kg}$ & $<5 \%$ \\
0,5 a $0,8 \mathrm{mg} / \mathrm{kg}$ & $10 \mathrm{a} 50 \%$ \\
$>0,8 \mathrm{mg} / \mathrm{kg}$ & $80 \%$ \\
\hline
\end{tabular}

FONTE: MEGARVANE (2000)

\section{RELATO DE CASO}

B. I., de 67 anos, foi internado em um hospital apresentando dor abdominal, vômitos e diarréia intensa. Após 4 horas de internamento constatou-se o óbito. Os exames hematológicos realizados no hospital apresentaram-se sem alterações. Após intensa investigação do médico sanitarista, constatou-se que a vítima havia começado tratamento com cápsulas manipuladas contendo o princípio ativo "Colchicina". Foram realizadas análises por cromatografia líquida de alta resolução (HPLC) nas cápsulas gelatinosas, fragmentos de fígado e rins. O procedimento analítico não ocorreu logo após o óbito, pois, a primeira suspeita da causa mortis foi hantavirose, ficando o material biológico armazenado por várias semanas, até que as investigações direcionaram o caso para intoxicação por colchicina.

O resultado da necrópsia apresentou placas hemorrágicas em toda a extensão do intestino delgado; fígado com bordos arredondados indicando congestão passiva; derrame pleural seroso, tendo $300 \mathrm{ml}$ no lado esquerdo e $350 \mathrm{ml}$ do lado direito, com sinais de pleurite aguda; edema e congestão pulmonar; estômago com focos hemorrágicos; edema cerebral; enterite hemorrágica; rins com congestão e petéquias nas regiões córtico medular. As análises anatomo patológicas indicaram: edema pulmonar; pielonefrite crônica focal; esteatose hepática, necrose hepática multifocal; edema e congestão cerebral.

\section{MATERIAL E MÉTODOS}

\subsection{Materiais}

a - 16 Cápsulas gelatinosas com 60mg de colchicina cada, de acordo com o rótulo da embalagem.

b - material biológico - fragmentos de fígado e rins

C - Equipamentos:

Sistema HPLC - Merck-Hitachi com detector DAD, monitoramento em 254 e 350 nm. Coluna Lichrospher 100 RP 18 (Merck 250 C 2,0 mm); temperatura do forno de $26^{\circ} \mathrm{C}$; fase móvel: Álcool Metílico, Acetonitrila e Tampão $\mathrm{KH}_{2} \mathrm{PO}_{4} 0,01 \mathrm{M}(\mathrm{pH} 7,6-41: 15: 44 \mathrm{v} / \mathrm{v} / \mathrm{V} /)$ isocrática, fluxo 0,8ml/min.

CG/MS - Finnigan Polaris Q; coluna de polidimetilsiloxane 5\% de fenila, temperatura programada de $60^{\circ} \mathrm{C} / 1 \mathrm{~min}, 20^{\circ} \mathrm{C} / \mathrm{min}$ até $300^{\circ} \mathrm{C} / 10 \mathrm{~min}$; injetor $200^{\circ} \mathrm{C}$, fluxo de He $1 \mathrm{ml} / \mathrm{min}$; temperatura da fonte $200^{\circ} \mathrm{C}$, transfer line $275^{\circ} \mathrm{C}$, SCAN 50-600 uma; injeção $1 \mathrm{ml}$ splitless 
d - Reagentes:

Metanol, acetonitrila e diclorometano grau HPLC - Merck, dihidrogênio fosfato monopotássico $\left(\mathrm{KH}_{2} \mathrm{PO}_{4}\right)$ - PA e monohidrogênio fosfato di-amônio $\left[\left(\mathrm{NH}_{4}\right)_{2} \mathrm{HPO}_{4}\right] \mathrm{PA}$ - Merck.

\subsection{Procedimento Analítico}

\subsubsection{Análise das cápsulas}

Doze cápsulas gelatinosas foram abertas, misturado seu conteúdo e pesado. O material foi diluído em metanol para se obter uma concentração aproximada de $1 \mathrm{mg} / \mathrm{ml}$. A solução obtida foi novamente diluida (1:100) com a fase móvel e analisada por HPLC.

A colchicina foi quantificada por padronização externa. Os pontos da curva foram de 500, 750, 1000, 1250, 1500 e 1750ng/ml, preparados em solução metanólica, conforme resultados na curva de calibração (figura 1).

\subsubsection{Análise do material biológico:}

9,5g de rim, homogeneizado em Turrax (1 parte de tecido e 3 partes de água); adicionado de $3 \mathrm{ml}$ de tampão fosfato $\mathrm{pH} 8\left[\left(\mathrm{NH}_{4}\right)_{2} \mathrm{HPO}_{4}\right]$ e $10 \mathrm{ml}$ de diclorometano para cada $3 \mathrm{~g}$ de tecido. Agitado, centrifugado, removida a fase orgânica e evaporada à secura. Redissolvido com $100 \mathrm{ml}$ de fase móvel e injetado $75 \mathrm{ml}$ no HPLC para análise. O mesmo procedimento foi usado para o fígado (KINTZ et al. 1997).

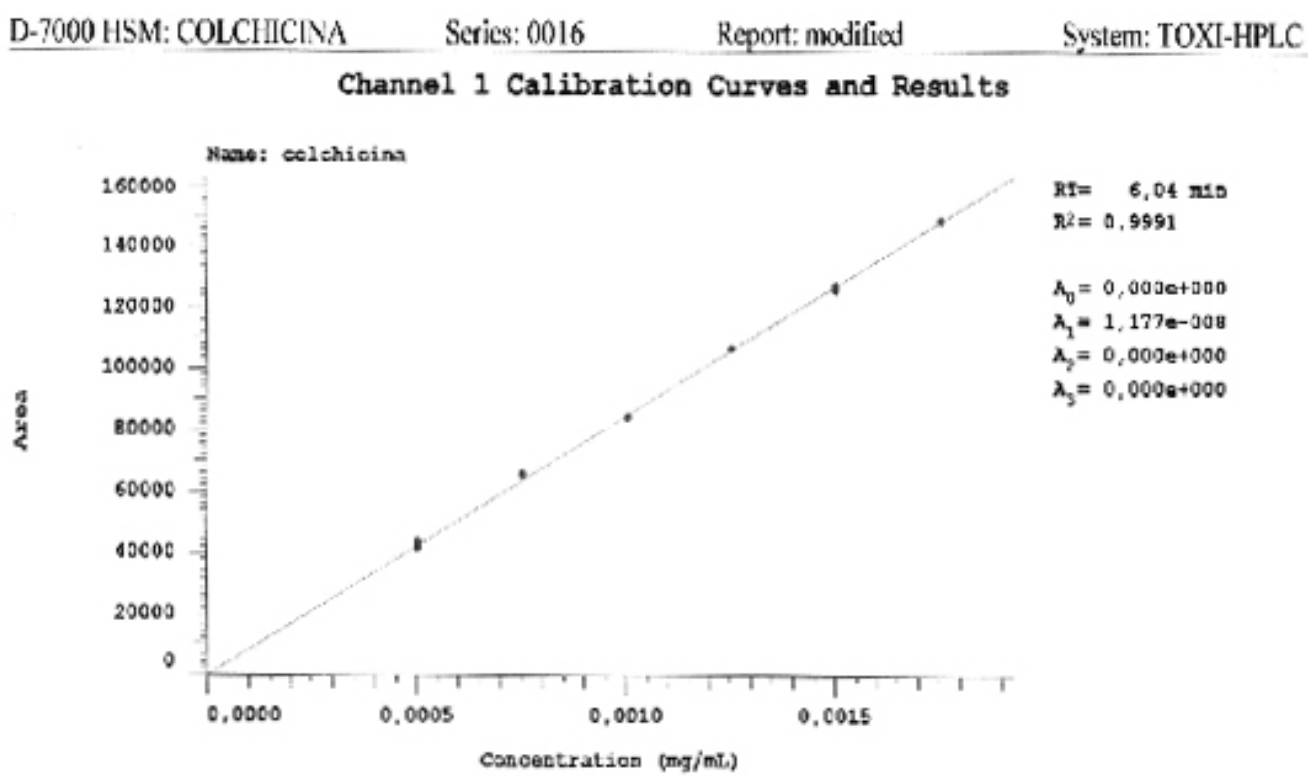

Chrom Type: Fixed WL Chromatcgram, $259 \mathrm{~nm}$

\begin{tabular}{|c|c|c|c|c|c|c|c|}
\hline Sane & Pts & Ao & A1 & 2) & $\mathrm{A}$ & $R-835$ & Units \\
\hline colchicinz. & $: 2$ & $0.0002-00$ & $1,1772-03$ & $0,000 \mathrm{z}-\mathrm{c}, 0$ & $0,000 E+00$ & $9,991 \mathrm{E}-01$ & nginL \\
\hline
\end{tabular}

Least Squazes Analysis

Drcez: Linear - fiResponse)

Through zero: YES

Weight factor: 1 . 0

FIGURA 1: CURVA DE CALIBRAÇÃO DA SOLUÇÃO PADRÃO DA COLCHICINA 


\section{RESULTADOS E DISCUSSÃO}

Nas cápsulas gelatinosas analisadas foi comprovada a presença da colchicina em concentração média de 63,48mg, valor próximo aquele expresso no rótulo da embalagem das cápsulas - 60mg/cápsula. As dosagens foram determinadas nas condições analíticas descritas acima, a colchicina eluiu em 6,09min (figura 2) e foi detectada a 350nm utilizandose detector de arranjo de diiodo (DAD) figura 3.

Chrom Type: Eixed wi Chromatogram, $350 \mathrm{~mm}$

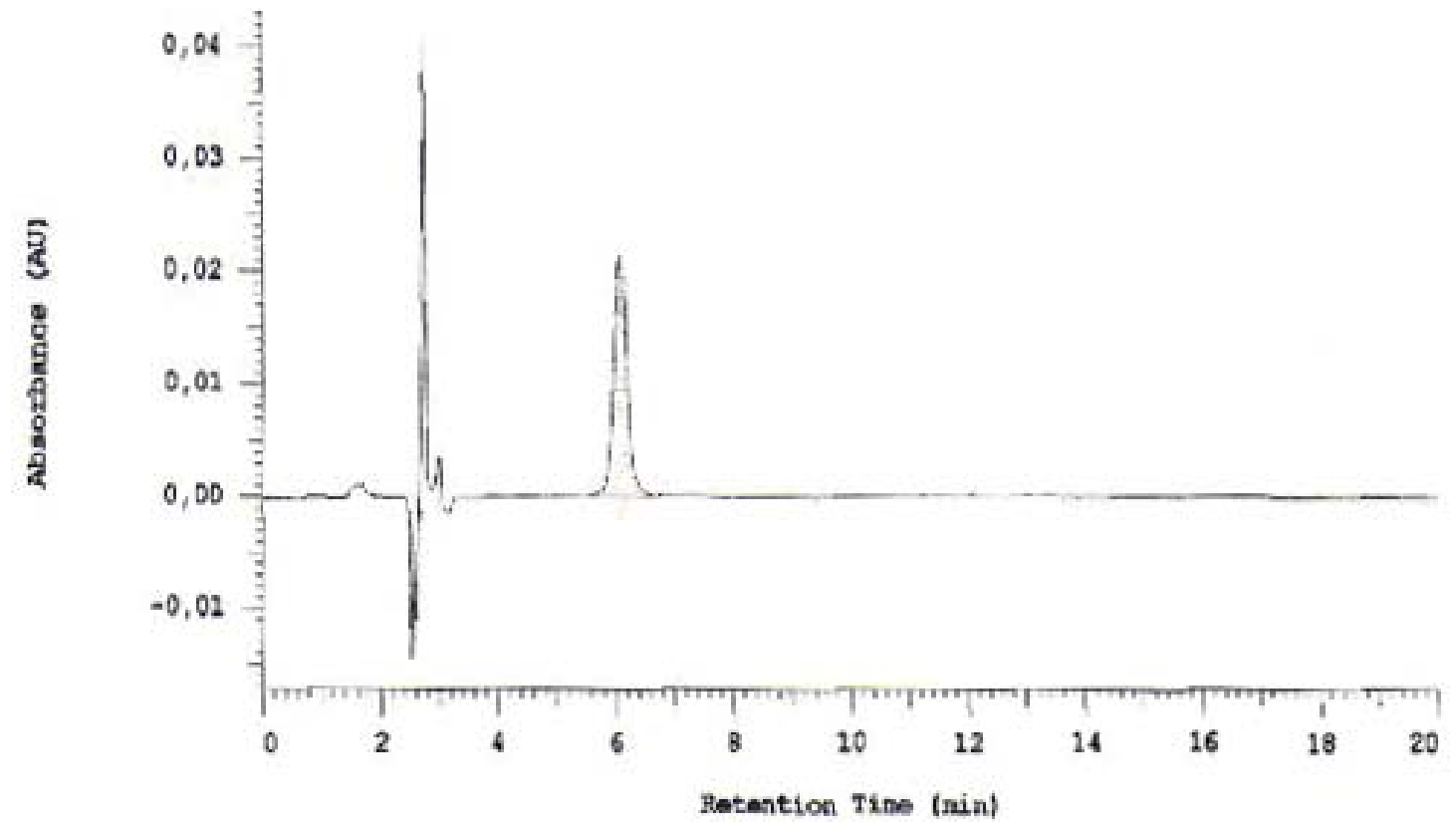

FIGURA 2: CROMATOGRAMA DA COLCHICINA ELUIDA EM 6,09MIN

D-7000 HSM:COLCHICINA Series:0019 Repon: modified Systant: TOXI-HPL.C

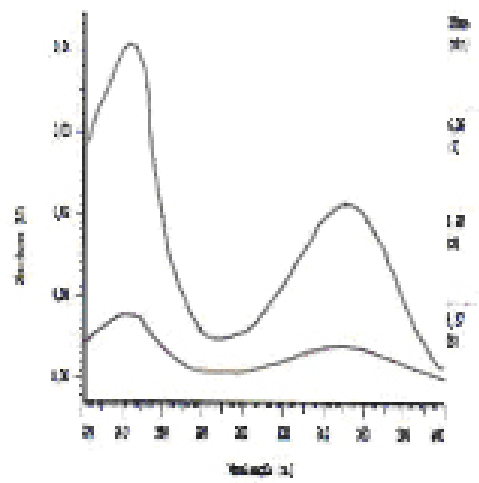

\begin{tabular}{|c|c|c|c|c|c|c|c|}
\hline No. & $\begin{array}{c}\text { Chrom } \\
\text { RT }\end{array}$ & Purity & Est. Cone & Corr & Speczear. & $\begin{array}{c}\text { Spectzum } \\
\text { RT }\end{array}$ & RIX \\
\hline 1 & 6.08 & $C, 3997$ & 1,37 & $\begin{array}{l}0.9997 \\
0,9696 \\
0,8 B 18\end{array}$ & $\begin{array}{l}\text { Coichicina } \\
\text { Indeno }[1,2,3 \text {-cid)pyrene }\end{array}$ & $\begin{array}{r}6,23 \\
16,92 \\
33,36\end{array}$ & $\begin{array}{l}0,00 \\
0,00 \\
0,00\end{array}$ \\
\hline
\end{tabular}

FIGURA 3: ESPECTRO DE ABSORÇÃO DA COLCHICINA OBTIDO PELO DETECTOR DE ARRANJO DE DIIODO 
A dose máxima diária indicada, ainda que varie entre os autores, é de $5 \mathrm{mg}$ (LUCAS, 1956; BATISTUZZA et al, 2000) e de 8mg (GOLDFRANK et al, 1994; MEGARVANE, 2000), tendo como indicação um intervalo de 3 dias antes de iniciar novo tratamento (LEIKIN et al, 1998).

No material biológico (rim e fígado) os resultados das análises de colchicina realizadas por HPLC e CG/MS foram negativos. Tais resultados talvez possam ser justificados pelo tipo de material biológico disponível, pois, de acordo com a literatura a bile é o material de escolha para análise da colchicina principalmente, em investigações pós morte (MOFFAT, 1986; KINTZ et. al, 1997; COFRAG, 2003). Outro fator que contribuiu para o resultado negativo, foi o tempo prolongado de armazenamento; uma vez que a colchicina em solução e em meio biológico degrada rapidamente em função de tempo, luz e temperatura (COFRAG, 2003).

\section{CONCLUSÃO}

A dosagem determinada de colchicina nas cápsulas analisadas foi superior ao que estava registrado no rótulo da embalagem e esta concentração é superior a dose terapêutica diária indicada pela literatura.

Apesar dos resultados negativos para colchicina obtidos no material biológico, houve a intoxicação fatal por overdose de colchicina estabelecida pelas evidencias: da necropsia, dos exames anatomo patológicos, sintomatologia da vítima no período de internação e pela concentração da colchicina nas cápsulas $(63,48 \mathrm{mg})$, dose equivalente a mais de 100 vezes a dosagem terapêutica indicada.

\section{REFERÊNCIAS}

GOLDFRANK, L.R. et al; Goldefrank's Toxicologic Emergencies; Appleton \& Lange - Norwalk,Connecticut, 1994. p. 671

KINTZ, P.; JAMEY, C.; TRACQUI, A.; MANGIN, P. Colchicine poisoning: Report of a fatal case and presentation of na HPLC procedure for body fluid and tissue analyses. JATOX 21, jan/ fev 1997. p. 70-72

ELLENHORN, M.J.; Ellenhorn's Medical Toxicology. 2 ed. Williams \& Williams: Baltimore, 1997. p. 1851-2

GONZÁLEZ ANGLADA, Ma. I.; ALONSO, S. J.; PUEYO, R. C.; MARA, J. C. e TORRES, E. Envenenamento por colchicina: uma intoxication grave poco conocida. Emergências, 10:5 sept/ oct 1998 p. $325-326$.

BATISTUZZO, J.A.O.; ITAYA, M. \& ETO, Y. Formulário Médico-Farmacêutico. São Paulo: Tecnopress, 2000. P. 110. REYNOLDS, J.E.F. (ed). Martindale the extra pharmacopoeia. 29 ed. London: The pharmaceutical Press, 1989. BAUD, F. J.; SABOURAUD, A.; VICAUT, E.; TABOULET, P.; LANG, J.; BISMUTH, C.; ROUZIOUX, J. M. \& SCHERRMANN, J. M. Brief Report: Treatament of severe colchicine overdose with colchicine-specific fab- Fragmentes. The New England Journal of Medicine, 332:10. March, 1995 p. 642-645

MEGARVANE, B. Intoxication aigue par la colchicine. Encyclopédie Orphanet, nov. 2000. http:// www.orpha.net/ data/ patho/ FR/ fr - colchicine.html. acessado 2003

LEIKIN, J.B.\& PALOUCEK, F.P.. Poisoning \& Toxicology Compendium. Lexi-Comp Inc: Ohio, 1998. p. 205-6

DANEL, V.C.; WIART, J.F; HARDY, G.A; VINCENT, F.H.; HOUDRET, N.M. Self-poisoning with Colchicum autumnale L. flowers. J.Forensic Sci, 39(1), Jan, 1994. p. 280-6 (10)

MOFFAT, A.C. at al.; Clarke's Isolation and identification of drugs, London:The Pharmaceutical Press, 1986. p. 492-3

LUCAS, V. Dicionários de Sinônimos. Rio: Cientif, 1956. p. 227.

COFRAG - Comité Français d'Accreditation da Sociedade Francesa. Ficha Técnica de Toxicologia Analítica Tox-230. Disponível em: < http://www.sfta.org/comission/COFRAC168/TOX230medleg.pdf > acessado em 2003. 